\title{
Trends in TRECs values according to age and gender in Chinese children, and their clinical application
}

\author{
Qin Zhao ${ }^{1}$ and Xiao-Dong Zhao ${ }^{1}$ \\ ${ }^{1}$ Children's Hospital of Chongqing Medical University
}

January 18,2021

\begin{abstract}
$\mathrm{T}$ cell receptor excision circles (TRECs) are small circularized DNA elements produced during rearrangement of $\mathrm{T}$ cell receptor (TCR) genes. Because TRECS are fairly stable, do not replicate during mitosis, and are not diluted during division of naïve $\mathrm{T}$ cells1, they are suitable for assessing the number of newly formed $\mathrm{T}$ cells 2 . In this study, we detected TRECs in 475 healthy Chinese children aged 0-18 years in different clinical settings. We found a strong correlation between TRECs levels and peripheral CD4 naïve T cell numbers, but not between TRECs levels and effector or memory CD4 and CD8 T cell numbers. TRECs levels fell significantly compared with normal controls in patients with severe combined immunodeficiencies (SCID) $(n=7)$, wiskott-aldrich syndrome (WAS) $(n=22)$, or activated PI3K $\delta$ syndrome (APDS) $(n=5)$. TRECs levels in those with signal transducer and activator of transcription 1 (STAT1) deficiency $(n=8)$ decreased or did not change significantly, a finding consistent with that for CD4 naïve T cells. We also measured TRECs levels in seven PIDs after hematopoietic stem cell transplantation $(\mathrm{HSCT})(\mathrm{WAS}=5$; chronic granulomatous disease $(\mathrm{CGD})=2)$, and found the complications after HSCT may reduce TRECs levels by interfering with production of naïve T cells. In conclusion, we established reference values for TRECs, which can be used to screen for primary immunodeficiency diseases (PIDs) during early life and track immune reconstitution after HSCT.
\end{abstract}

Trends in TRECs values according to age and gender in Chinese children, and their clinical application

Qin Zhao ${ }^{1,2}$, Rongxin Dai ${ }^{1,2,3}$, Yanan $\mathrm{Li}^{1,2}$, Yanping Wang ${ }^{1,2}$, Xuemei Chen ${ }^{1,2}$, Zhou Shu ${ }^{1,2,3}$, Lina Zhou ${ }^{1,2}$, Yuan Ding ${ }^{1,2,4}$, Xuemei Tang ${ }^{3}$, Xiaodong Zhao ${ }^{1,2,3^{*}}$

1. Department of Pediatric Research Institute; Ministry of Education Key Laboratory of Child Development and Disorders; National Clinical Research Center for Child Health and Disorders (Chongqing); China International Science and Technology

Cooperation base of Child development and Critical Disorders, Children's Hospital of Chongqing Medical University, Chongqing, China

2. Chongqing Key Laboratory of Child Infection and Immunity, Children's Hospital of Chongqing Medical University, Chongqing China, 400014.

3. Department of Rheumatology and Immunology, Children's Hospital of Chongqing Medical University, Chongqing China, 400014.

4. Department of Health Management, Children's Hospital of Chongqing Medical University, Chongqing China, 400014

\section{Corresponding author:}

Xiaodong Zhao 
Children's Hospital of Chongqing Medical University, No. 136, Zhongshan 2nd Road, Yuzhong District, Chongqing China, 400014

Tel: +86-23-63630602; Fax: +86-23-63602136

E-mail: zhaoxd530@aliyun.com

\section{Abstract}

T cell receptor excision circles (TRECs) are small circularized DNA elements produced during rearrangement of $\mathrm{T}$ cell receptor (TCR) genes. Because TRECS are fairly stable, do not replicate during mitosis, and are not diluted during division of naïve $\mathrm{T}$ cells ${ }^{1}$, they are suitable for assessing the number of newly formed $\mathrm{T}$ cells $^{2}$. In this study, we detected TRECs in 475 healthy Chinese children aged 0-18 years in different clinical settings. We found a strong correlation between TRECs levels and peripheral CD4 naïve T cell numbers, but not between TRECs levels and effector or memory CD4 and CD8 T cell numbers. TRECs levels fell significantly compared with normal controls in patients with severe combined immunodeficiencies (SCID) $(\mathrm{n}=7)$, wiskott-aldrich syndrome (WAS) $(\mathrm{n}=22)$, or activated PI3Ko syndrome (APDS) $(\mathrm{n}=5)$. TRECs levels in those with signal transducer and activator of transcription 1 (STAT1) deficiency $(n=8)$ decreased or did not change significantly, a finding consistent with that for CD4 naïve T cells. We also measured TRECs levels in seven PIDs after hematopoietic stem cell transplantation (HSCT) (WAS $=5$; chronic granulomatous disease $(C G D)=2$ ), and found the complications after HSCT may reduce TRECs levels by interfering with production of naïve T cells. In conclusion, we established reference values for TRECs, which can be used to screen for primary immunodeficiency diseases (PIDs) during early life and track immune reconstitution after HSCT.

\section{Key words: TRECs; reference values; PIDs; HSCT}

\section{Introduction}

The thymus provides a suitable microenvironment for maturation of $\mathrm{T}$ cells; therefore, thymic output may reflect thymus function. $\mathrm{T}$ cell receptor excision circles (TRECs) are circular DNA segments generated in T cells during sequential rearrangement of the variable V, D, and J segments of TCR genes. About $70 \%$ of all newly produced $\mathrm{T}$ cells are TREC-positive. These circularized DNA elements cannot replicate or be stored in the cells ${ }^{3}$. Thus, quantitation of TRECs is an excellent surrogate marker of the number of naïve $\mathrm{T}$ cells that have emigrated recently from the thymus ${ }^{4}$.

Since measurement of TRECs can be done quickly by real-time quantitative PCR (RT-qPCR), TRECs levels have been used to assess thymic output under healthy and disease conditions; they are especially useful for diagnosis and management of $\mathrm{T}$ cell-related disorders ${ }^{5}$. In recent years, TRECs have been used to screen newborns for $\mathrm{SCID}^{6}$. Low TRECs levels have been detected in preterm newborns and low birth weight (BW) babies. Newborns with Down's syndrome and ataxia telangiectasia have low TRECs levels ${ }^{7,8}$.

As TRECs are a marker of $\mathrm{T}$ cell reconstitution, levels should predict occurrence of GVHD after HSCT. Indeed, patients with low TRECs levels after HSCT are more likely to suffer GVHD ${ }^{9,10}$. Assessing trends in TRECs levels according to age is used as a forensic investigation tool to estimate age ${ }^{11}$. TRECs levels could be used to distinguish between benign and malignant diseases; indeed, studies show that levels in patients with acute lymphocytic leukemia and acute myeloid leukemia are lower than those in healthy persons ${ }^{12}$. In addition, TRECs analysis has been applied to autoimmune diseases. TRECs levels in patients with systemic lupus erythematosus fall as disease activity increases ${ }^{13}$. Levels are also low in patients with autoimmune thyroiditis. However, levels in those with autoimmune type 1 diabetes are higher than those in healthy controls ${ }^{14}$.

Several studies have examined thymic function in healthy children and adults ${ }^{15,16}$. However, in China, we have no reference values for TRECs in different pediatric age groups. Therefore, it is difficult to determine a cut-off value for TRECs in a clinical setting. Also, it is not completely clear which diseases impact TRECs values. Here, we examined trends in TRECs levels in 475 healthy children (aged 0-18 years) according to 
age; the cohort included premature newborns and low BW newborns. Combined with analysis of lymphocyte subsets, we demonstrated a strong correlation between TRECs levels and CD4 naïve T cell numbers. To test the significance of these findings in a clinical setting, we evaluated TRECs levels in patients with different PIDs and patients with PIDs treated with HSCT.

\section{Methods}

\section{Healthy volunteers}

A total of 410 healthy children (227 males, 183 females) aged 0-18 years were recruited. All were healthy volunteers from Chongqing, China. We defined "healthy" as follows: normal nutritional status, no evidence of infectious disease, no medication, no contact with persons with infectious disease, no immunizations for at least the last 4 weeks, no blood transfusions, no history of atopic disorders, and no evidence of hematological or immunological disorders. All participants underwent regular blood tests, liver and renal function tests, serological tests for hepatitis B virus, and real-time PCR for EBV and cytomegalovirus. Blood samples were obtained from September 2014 to June 2015. DNA was extracted from peripheral blood.

\section{Dry blood spots sample collection}

Whole blood samples $(\mathrm{n}=65)$ were dropped onto Whatman 903 paper in accordance with the established routine neonatal screening process. Three $3.2 \mathrm{~mm}$ discs were punched per sample and stored at $4 * \mathrm{C}$ until they were processed. DNA was extracted according to a standard protocol (QIAGEN).

\section{Patients}

The volunteers recruited to the study were aged from 0-18 years; all were from nonconsanguineous families in mainland China, and included premature newborns and low BW newborns. Those with PIDs were admitted to the Children's Hospital of Chongqing Medical University and diagnosed according to clinical features, laboratory inspection, and genetic analysis. WAS or CGD patients accepted for transplantation were followed up at Children's Hospital of Chongqing Medical University. Clinical data were collected during hospitalization. Informed consent was obtained from participants or their guardians.

\section{Quantification of TRECs}

Genomic DNA was isolated from peripheral blood samples according to standard protocols (QIAGEN). The sequences of the primers used for PCR were as follows: TRECs, 5'-CACATCCCTTTCAACCATGCT-3' and 5'-GCCAGCTGCAGGGTTTA GG-3'; probe, 5'-FAM-ACACCTCTG GTTTTTGTAAAGGTGCCCACT3'-TAMRA; and T cell receptor alpha constant sequences, 5'-AGGAATCCTTGTCTCTGAAAAA TGC3' and 5'-TTCCTTTTAGTTTCTTGGCCTATGC-3'; probe, 5'-HEX-TGAAGA GAGGACCCTGTTACCGCC A-3'-TAMRA. TRECs were measured by real-time quantitative PCR, as described previously. Briefly, $5 \mu \mathrm{l}$ of DNA was amplified in $25 \mu \mathrm{l}$ of PCR solution containing primers and probes specific for TRECs. Standards were prepared according to protocols published by Sottini et $\mathrm{al}^{17}$. All DNA samples were run in triplicate alongside no-template controls.

\section{Lymphocyte subset analysis}

All the healthy volunteers were taking part in the Public Welfare Scientific Research Project of China (grant no. 201402012) ${ }^{18}$. The BD Multi-test IMK Kit (cat. 340503) was used to analyze lymphocyte subsets (B cells, CD3 T cells, CD4 T cells, CD8 T cells, and CD32CD56/CD16 natural killer cells). The following mAbs were used to detect T cell subsets: anti-human CD3 (PerCP-Cy5.5), anti-CD4 (FITC; fluorescein isothiocyanate), anti-CD8 (BV510), anti-CD27 (APC; allophycocyanin), anti-CD45RA (PE-Cy7), anti-TCR (PE; phycoerythrin), and anti-TCRgd (BV421) (all from BD Biosciences). Subsets were analyzed as described previously.

\section{Statistical analysis}

Data were analyzed using GraphPad prism software and the IBM SPSS Statistics package (version 20). Independent-samples t-tests were used to compare the means of two groups (parametric data). Pearson's 
and partial correlation coefficients were calculated for parametric and nonparametric data, respectively. A $\mathrm{P}$-value $<0.05$ was considered statistically significant.

\section{Results}

\section{Trends in TRECs levels in healthy children according to age and gender}

We hypothesized that understanding trends in TRECs levels in healthy children according to age will help us to identify disease at the early stages; therefore, we quantified TRECs levels in 475 healthy children, including premature newborns and low weight newborns. In accordance with a previously published paper ${ }^{18}$, the children were categorized into seven different age groups (0-1 months $(\mathrm{m}), 1 \mathrm{~m}-6 \mathrm{~m}, 6 \mathrm{~m}-1$ years $(\mathrm{y})$, 1-4 y, 4-8 y, 8-12 y, and 12-18 y) (Table 1). TRECs levels fell significantly with increasing age ( $\mathrm{r}-0.5179$; $\mathrm{p}<0.0001$ ) (Fig. 1), which is consistent with the findings of $\mathrm{Ou}$ et $\mathrm{al}^{19}$. TRECs declined rapidly from $1 \mathrm{~m}$ to $4 \mathrm{y}$ and from $12 \mathrm{y}$ to $18 \mathrm{y}$. There were no obvious changes between newborns and babies aged 1-6 $\mathrm{m}$. Females tended to have higher levels of TRECs than males before the age of 1 year; however, the difference was not statistically significant. After the age of 1 year, TRECs levels in females were similar to those in males (Fig. 2).

We also analyzed TRECs levels according to gestation age (GA) (Fig. 3A). TRECs levels increased with the increasing GA. Levels in preterm infants were significantly lower than in full term babies (Fig. 3B), which is consistent with the findings of de Felipe et $\mathrm{al}^{20}$. Here, we found that full term newborns with low BW had lower TRECs levels than those of normal BW (Fig. 3C). However, the TRECs levels in preterm infants with low BW were comparable with those of normal BW infants (Fig. 3D).

\section{TRECs levels correlate significantly with CD4 naïve T cell numbers}

To examine the correlation between $\mathrm{T}$ cell subsets and TRECs levels, we analyzed the absolute numbers of different $\mathrm{T}$ cell subsets in the 410 healthy children. There was a correlation between TRECs levels and the numbers of CD3 T cells (r 0.220, p<0.0001) (Fig. 4A) and CD4 T cells (r 0.318, p<0.0001) (Fig. 4B). We also found a significant correlation between TRECs levels and CD4+CD45RA+CD27+ (CD4 naïve) $\mathrm{T}$ cell numbers (r 0.305, p<0.0001) (Fig. 4C). However, there was no correlation between TRECs levels and CD8+/CD8+CD45RA+CD27+ (CD8 naïve) T cell numbers ( $\mathrm{p}>0.1)($ Fig. 4D/E), or between TRECs levels and $\mathrm{CD} 4+/ \mathrm{CD} 8+$ central memory $\mathrm{T}$ cell or CD4/CD8 effector memory $\mathrm{T}$ cell numbers (Fig. 4F-H).

Because CD4 naïve T cell numbers showed a strong correlation with TRECs levels, we next analyzed the correlation between TRECs levels and CD4 naïve T cell numbers in different age groups (Fig. 5). We found significant correlations between TRECs numbers and CD4 naïve T cell numbers in the $1-4$ y $(\mathrm{r} 0.243, \mathrm{p}$ 0.023) (Fig. 5D) and $4-8$ y (r 0.409, p<0.0001) age groups (Fig. 5E).

\section{TRECs levels in children with PIDs and secondary immunodeficiency}

To confirm the specificity and sensitivity of our method, we analyzed TRECs levels in patients with SCID and X linked agammaglobulinemia (XLA). TRECs levels in patients with SCID of the IL2rg, RAG1, JAK3, and Lig4 mutation genotypes were 0 , whereas those in patients with XLA were normal (Table 1), which is consistent with previous publications ${ }^{21}$.

Next, we determined the significance of TRECs levels in PIDs (Table 2). We identified greater reductions in TRECs levels in patients with classical WAS, whereas those in X linked thrombocytopenia (XLT) patients were only slightly decreased or within the normal ranges (Fig. 6). These data suggest that TRECs levels could be used to identify classical WAS or XLT early, thereby enabling appropriate selection of therapeutics. However, the data may mean that classical WAS might be caused by a thymic output defect ${ }^{22}$. Gain-offunction (GOF) mutations in PIK3CD cause APDS. Therefore, we asked whether there was a clear reduction in TRECs levels in APDS, consistent with other combined immunodeficiency (CIDs). We found that TRECs levels in most patients with STAT1 mutations were normal, although some did have lower levels (Fig. 6). We also analyzed the lymphocyte subsets from STAT1 patients (Table 3) and found that changes in TRECs levels were consistent with changes in CD4 naïve T cell numbers in STAT1 patients (Fig. 7). 
In addition, we analyzed TRECs levels in patients with secondary immunodeficiencies such as primary nephrotic syndrome (NS) after prednisone treatment. We found that TRECs levels were normal in NS patients with or without immunosuppressive treatment (Table 4 and Fig. 8).

\section{TRECs levels in PID patients after HSCT}

We followed up TRECs levels in five patients with classical WAS and two with CGD who underwent HSCT (Fig. 9). The clinical features of these patients are summarized in Table 5. Patients P2 and P5 characterized by respiratory tract infections, eczema, and thrombocytopenia. P2 developed GVHD in the liver, eyes, and CNS after HSCT, and P5 suffered multiple organs disorders. P6 on set with systemic tuberculosis after BCG vaccination and suffered liver and kidney damage after HSCT. P2, P5, and P6 had low levels of TRECs up until 4 weeks after HSCT. The other four patients showed elevated TRECs levels in the following 4 weeks, which returned to the normal range at around 1 year post-HSCT.

\section{Discussion}

TRECs are thought to be the most reliable tools for tracking recent thymic output. Indeed, such measurements have been used in multiple clinical settings, including diagnosis of SCID, T cell development-associated immune deficiencies, T cell reconstitution after HSCT, aging, and autoimmune diseases.

In this study, we quantified TRECs levels in 475 children aged 0-18 years, including premature newborns and low BW newborns. To the best of our knowledge, this is the largest study designed to determine TRECs reference levels in a healthy Chinese pediatric population. We found that TRECs levels tended to fall with age, mainly due to division of peripheral cells and reduced thymic activity due to age-associated thymic involution $^{23}$. TRECs levels fell rapidly between 1 month and 1 year-of-age, reflecting increased rates of thymopoiesis. A second large decrease occurred from 12-18 years-of-age, which might be due to changes in hormone levels. We also found that TRECs levels were lower in premature newborns and low BW newborns. TRECs levels tended to increase with GA. These data suggest that during newborn screening, the GA and its reciprocal BW should be included in the screening strategies ${ }^{24}$. Ward et al found a $9.8 \%$ increase in TRECs levels per week of gestation ${ }^{25}$; however, our study identified no linear correlation between GA and TRECs levels, although this may be due to an insufficient number of subjects in this case.

Gender-related differences in TRECs levels were very conflicting ${ }^{26,27}$. We found that TRECs levels in females were similar to those in males. Sex hormones such as prolactin may regulate development of CD4 T cells ${ }^{28}$. Indeed, the number of mature CD4 T cells in estrogen-treated mice increases. Also, testosterone may induce apoptosis of thymocytes ${ }^{29}$. In addition, production of cytokines is affected by sex hormones such as IL7 and IL15, which may drive development of $\mathrm{T}$ cells ${ }^{30}$.

We found a weak correlation between TRECs levels and numbers of CD4 naïve T cells, which is inconsistent with the consensus that TRECs levels are closely related to CD4 naïve T cell numbers. In the large number of healthy young participants, we found a correlation between TRECs levels and the absolute number of peripheral CD4 naïve T cells only in the 1-4 y and 4-8 y age groups; no study has found this before. This finding may be due to the fact that we examined CD4 naïve T cell numbers in peripheral blood; thus the numbers may be affected by factors other than thymic output (i.e., proliferation, death, and redistribution of peripheral CD4 naïve T cells) ${ }^{31}$. The CD4 naïve $\mathrm{T}$ cells have a short lifespan and soon undergo apoptosis, thereby contributing to $\mathrm{T}$ cell homeostasis in which newly generated thymic emigrants make up for the loss of peripheral cells ${ }^{32}$. Activated of CD4 naïve T cells are regulated by some nutritional factors ${ }^{33}$; therefore, measurement of TRECs levels and naïve T cell numbers should be combined with an assessment of thymus function.

WAS, characterized by eczema, thrombo-cytopenia, and immunodeficiency, has three phenotypes: classical WAS, XLT, and X-linked neutropenia (XLN) ${ }^{34}$. Treatment differs according to the phenotype and associated complications. Those with classical WAS need urgent curative treatment, such as a stem cell transplant or gene therapy ${ }^{35}$. For those with XLT, symptomatic treatment is the major therapy of choice, although splenectomy is sometimes recommended as it effectively stops the tendency to bleed ${ }^{36}$. XLT patients have 
a better prognosis than those with WAS ${ }^{37}$. Thus, WAS may be a good model to test whether TRECs are a good biomarker and predictor of disease severity. Our data revealed that TRECs levels in patients with classical WAS $(\mathrm{n}=14)$ were significantly suppressed, whereas those in patients with XLT $(\mathrm{n}=8)$ were mildly suppressed or normal. There was a strong correlation between TRECs levels and CD4 naïve cell numbers in those with classic WAS, which is consistent with the findings of a previous study ${ }^{36}$.

GOF mutations in the PI3K genes PIK3CD (p1106) and PIK3R1 (p85 $\alpha$ ) cause a combined immunodeficiency syndrome called APDS ${ }^{38}$. TRECs levels and CD4 naïve T cell numbers were suppressed significantly in those with APDS ${ }^{39}$. The PI3K-AKT pathway is crucial for transition of intermediate single positive thymocytes to double positive thymocytes ${ }^{40}$. T cell development in those with PIK3CD GOF mutations is skewed, as evidenced by a reduction in CD4 and CD8 thymocyte numbers in an APDS mouse model ${ }^{41}$. APDS mice show severe lymphopenia in the periphery, along with increased senescence of effector $\mathrm{T}$ cells and total $\mathrm{T}$ cells $^{42}$. Signal transducer and activator of transcription 1 (STAT1) is a transcription factor that mediates cellular responses to interferons (IFNs), as well as other cytokines and growth factors. Mutation of STAT1 is associated with chronic mucocutaneous candidiasis or Mendelian susceptibility to mycobacterial disease, with or without autoimmune disease ${ }^{43}$. TRECs levels in patients with STAT1 mutation are normal in the publications before44,45; however, we found that TRECs levels were consistent with the levels of CD4 naïve T cells, which were normal or mildly decreased. A previous study shows that only $28 \%$ of patients with STAT1 mutations have low CD4 $\mathrm{T}$ cell numbers ${ }^{46}$. We found a similar result here ${ }^{47}$. Lower CD4 T cell counts are associated with a higher mortality in STAT1 patients; therefore, patients with low TRECs levels probably require HSCT as soon as possible. The proportion of CD3/CD4 T cells and TRECs levels in NS patients were normal ${ }^{48,49}$. Overall, the data suggest that TRECs levels correlate with CD4 naïve T cell numbers; therefore, they may be a good biomarker for multiple PIDs.

TRECs levels may also predict occurrence of GVHD and associated complications. In the first few months after HSCT, peripheral expansion of implanted cells is very important ${ }^{50}$. We found that TRECs levels started to rise within 4 weeks of HSCT, and achieved normal levels by 1 year post-HSCT. Consistent with the findings of Weinberg et $\mathrm{al}^{51}$, we found that GVHD might be associated with low TRECs levels. P2 developed GVHD in multiple organs, and his TRECs levels remained low. P5 and P6 had low TRECs levels after HSCT and developed multiple organ disorders. The major factors that affect TRECs levels are the number of HLA matches, the type of graft, TRECs levels before HSCT, and the proposal conditioning regimen ${ }^{52}$. However, more cases are needed to identify a correlation between TRECs levels and complications after HSCT.

\section{Conclusions}

The present study used RT-qPCR to analyze TRECs levels in 475 healthy children of different ages in China. This is the largest population of healthy children to be analyzed in China. We established reference values for TRECs and quantified trends in TRECs levels according to age and sex. We provide solid data showing a correlation between CD4 naïve T cell counts and TRECs levels according to age. Postnatal factors such as nutrition and hormone levels induce rapid changes in the phenotype of CD4 naïve T cells. We also showed that TRECs levels are of clinical utility by quantifying them in patients with WAS, APDS, or STAT1 mutations, in patients post-HSCT, and in patients with secondary immunodeficiency disease. Although the methods used to measure and detect TRECs differ between laboratories (which will lead to differences in TRECs levels in the same individual), it is still meaningful to establish a TRECs reference value within a consistent environment. Our institution is the main PID diagnosis and treatment center in China; therefore, such a reference value system will enable prompt screening of PIDs in a timely manner.

\section{Acknowledgements}

We are grateful to all the healthy participants, the patients and their families for their continuous corporation in this study. We thank the members of the laboratory for their technical assistance. We thank doctors and nurses for their generous supporting this project.

\section{Ethics approval}


Informed consent was obtained from all individual participants included in the study. This study was conducted in accordance with the tenets of the Declaration of Helsinki and was approved by the ethics committee of Chongqing Medical University.

\section{Authorship Contributions}

Q.Z and ZX.D designed the study and wrote the manuscript; Q.Z, RX.D and YN.L performed the experiments and analyzed the data; Q.Z, YP.W, XM.C, Z.S, LN.Z, Y.D and XM.T followed the patients; all the authors reviewed the manuscript before it was submitted.

\section{Funding information}

This work was supported by the Science and Technology Research Program of Chongqing Municipal Education Commission (KJZD-M201800401) and Chongqing Postgraduate Research and Innovation Project (CYB18157).

\section{Consent to participate}

Written informed consent was obtained from individual or guardian participants.

\section{Consent to publication}

Written informed consent was obtained from individual or guardian participants.

\section{Availability of data and material}

The data that support the findings of this study are available from the corresponding authors, XD.Z upon reasonable request.

\section{Disclosure of Conflicts of Interest}

The authors declare no conflict of interest.

\section{Reference}

1.Dion ML, Se'kaly RP, Cheynier R. Estimating thymic function through quantification of T-cell receptor excision circles. Methods Mol Biol 2007, 380: 197 - 213.

2. Ping Ye and Denise E. Reevaluation of T Cell Receptor Excision Circles as a Measure of Human Recent Thymic Emigrants. The Journal of Immunology, 2002, 168(10):4968-79.

3. Marina Garcia-Prat, Daniel Álvarez-Sierra, Aina Aguilo-Cucurull. Extended Immunophenotyping Reference Values in a Healthy Pediatric Population. International Clinical Cytometry Society. 2018, 96(3):223233.

4. S. P. Adams, S Kricke, E Ralph. A comparison of TRECs and flow cytometry for naive T cell Quantification, Clinical and Experimental Immunology, 2017, 191(2):198-202.

5. Haynes BF, M L Markert, G D Sempowski, D D Patel. The role of the thymus in immune reconstitution in aging, bone marrow transplantation, and HIV-1 infection. Annu Rev Immunol 2000; 18:529-560.

6. Khyber Shinwari, Mikhail Bolkov, Irina A Tuzankina. Newborn Screening through TREC, TREC/KREC system for Primary Immunodeficiency with limitation of TREC/KREC. Antiinflamm Antiallergy Agents Med Chem. E-pub Ahead of Print 2020 Jul 30.

7. Verstegen, R. H. J, Stephan Borte, Levinus A Bok. Impact of Down syndrome on the performance of neonatal screening assays for severe primary immunodeficiency diseases. Journal of Allergy and Clinical Immunology, 2014, 133(4), 1208-1211.

8. Amarilla B Mandola, B Reid, R Sirror. Ataxia Telangiectasia Diagnosed on Newborn Screening Case Cohort of 5 Years' Experience. Front Immunol. 2019 Dec 20;10:2940. 
9. Maria V. Soares, Rita I Azevedo, Ines A Ferreira. Naive and Stem Cell Memory T Cell Subset Recovery Reveals Opposing Reconstitution Patterns in CD4 and CD8 T Cells in Chronic Graft vs. Host Disease. Front Immunol. 2019 Mar 6;10:334.

10. Gaballa, A, A Norberg, A Stikvoort. Assessment of TREC, KREC and telomere length in long-term survivors after allogeneic HSCT: the role of GvHD and graft source and evidence for telomere homeostasis in young recipients. Bone Marrow Transplant. 2018 Jan;53(1):69-77.

11.Eisuke Yamanoi, Saori Uchiyama, Makoto Sakurada. SjTREC quantification using SYBR quantitative PCR for age estimation of bloodstains in a Japanese population. Legal Medicine. 2018 May;32:71-74.

12. Neveen Lewis Mikhael, Manal Elsorady. Clinical significance of T cell receptor excision circle (TREC) quantitation after allogenic HSCT. BLOOD RESEARCH. 2019 Dec;54(4): 274-281.

13. Queila F Vieira, Cristiane Kayser, Esper G Kallas. Decreased Recent Thymus Emigrant Number Is Associated with Disease Activity in Systemic Lupus Erythematosus. J Rheumatol 2008;35;1762-1767.

14. Samrina Mahtab, Parmita Kar, Soma Saha. Central Immune Tolerance of T and B Cells in Patients With Idiopathic Hypoparathyroidism, T1D, and Autoimmune Thyroiditis. Endocrine Society. 2019 Feb $20 ; 3(6): 1175-1184$.

15. Marina Garcia-Prat, Daniel Alvarez-Sierra, Aina Aguilo-Cucurull. Extended Immunophenotyping Reference Values in a Healthy Pediatric Population. International Clinical Cytometry Society. 2019 May;96(3):223-233.

16. A.Levy, A Rangel-Santos, L C Torres. T cell receptor excision circles as a tool for evaluating thymic function in young children. Brazilian Journal of Medical and Biological Research. 2019; 52(7): e8292.

17. Alessandra Sottini, Federico Serana 1, Diego Bertoli. Simultaneous Quantification of T-Cell Receptor Excision Circles (TRECs) and K-Deleting Recombination Excision Circles (KRECs) by Real-time PCR. J. Vis. Exp. 2014 Dec 6;(94):52184.

18. Yuan Ding, Lina Zhou, Yu Xia. Reference values for peripheral blood lymphocyte subsets of healthy children in China. J Allergy Clin Immunol. 2018 Sep;142(3):970-973.

19. Xueling Ou, Hu Zhao, Hongyu Sun. Detection and quantification of the age-related sjTREC decline in human peripheral blood. Int J Legal Med. 2011 Jul;125(4):603-8.

20. de Felipe B, Peter Olbrich, Jose Manuel Lucenas. Prospective neonatal screening for severe T- and B-lymphocyte deficiencies in Seville. Pediatr Allergy Immunol. 2016 Feb;27(1):70-7.

21. Jet van der Spek, Rolf H H Groenwold, Mirjam van der Burg. TREC Based Newborn Screening for Severe Combined Immunodeficiency Disease: A Systematic Review. J Clin Immunol. 2015 May;35(4):416-30.

22. Wenyan Li, Xiaoyu Sun, Jinzhi Wang. Defective thymic output in WAS patients is associated with abnormal actin organization. Sci Rep. 2017 Sep 20;7(1):11978.

23. C M Steffens, L Al-Harthi, S Shott. Evaluation of Thymopoiesis Using T Cell Receptor Excision Circles (TRECs): Differential Correlation between Adult and Pediatric TRECs and Naive Phenotypes. Clin Immunol. 2000 Nov;97(2):95-101.

24. P. Olbrich, B de Felipe, C Delgado-Pecellin. A first pilot study on the neonatal screening of primary immunodeficiencies in Spain: TRECS and KRECS identify severe T- and B-cell lymphopaenia. An Pediatr (Barc). 2014 Nov;81(5):310-7.

25. Ward CE, Baptist AP. Challenges of newborn severe combined immune-

deficiency screening among premature infants. Pediatrics. 2013 Apr;131(4):e1298-302. 
26. J Pido-Lopez, N Imami, R Aspinall. Both age and gender affect thymic output: more recent thymic migrants in females than males as they age. Clin Exp Immunol. 2001 Sep;125(3):409-13.

27. Leila Shakerian, Zahra Pourpak, Somayeh Shamlou. Determining Laboratory Reference Values of TREC and KREC in Different Age Groups of Iranian Healthy Individuals. Iran J Allergy Asthma Immunol. 2019 Apr 1;18(2):143-152.

28. Elena Ortona, Marina Pierdominici, Angela Maselli. Sex-based differences in autoimmune diseases. Ann Ist Super Sanita. Apr-Jun 2016;52(2):205-12.

29. S A Ahmed, B D Hissong, D Verthelyi,c. Gender and Risk of Autoimmune Diseases: Possible Role of Estrogenic Compounds. Environ Health Perspect. 1999 Oct;107 Suppl 5(Suppl 5):681-6.

30. M. Pinti, M Nasi, E Lugli. T Cell Homeostasis in Centenarians: From the Thymus to the Periphery. Curr Pharm Des. 2010;16(6):597-603.

31. Ruy. Ribeiro, Alan S Perelson. Determining thymic output quantitatively: using models to interpret experimental T-cell receptor excision circle (TREC) data. Immunol Rev. 2007 Apr;216:21-34.

32. Hirotake Tsukamoto, Karen Clise-Dwyer, Gail E Huston. Age-associated increase in lifespan of naive CD4 T cells contributes to T-cell homeostasis but facilitates development of functional defects. Proc Natl Acad Sci U S A. 2009 Oct 27; 106(43): 18333-8.

33. Donte C Saucillo 1, Valerie A Gerriets, John Sheng. Leptin Metabolically Licenses T Cells for Activation to Link Nutrition and Immunity. J Immunol. 2014 Jan 1;192(1): 136-44.

34. Hans D Ochs, Alexandra H Filipovich, Paul Veys. Wiskott-Aldrich Syndrome: Diagnosis, Clinical and Laboratory Manifestations, and Treatment. Biol Blood Marrow Transplant. 2009 Jan;15(1 Suppl):84-90.

35. Austen J J Worth, Adrian J Thrasher. Current and emerging treatment options for Wiskott-Aldrich syndrome. Expert Rev Clin Immunol. 2015;11(9):1015-32.

36. Hans D Ochs, Adrian J Thrasher. The Wiskott-Aldrich syndrome. J Allergy Clin Immunol. 2006 Apr;117(4):725-38.

37. Michael H Albert, Tanja C Bittner, Hans D. Ochs. X-linked thrombocytopenia (XLT) due to WAS mutations: clinical characteristics, long-term outcome, and treatment options. Blood. 2010 Apr 22;115(16):32318.

38. Carrie L Lucas, Anita Chandra, Sergey Nejentsev. PI3Ko and primary immune-deficiencies. Nat Rev Immunol. 2016 Nov;16(11):702-714.

39. Yanping Wang, Xuemei Chen, Qiuyun Yang. E1021K Homozygous Mutation in PIK3CD Leads to Activated PI3K-Delta Syndrome 1. J Clin Immunol. 2020 Feb; 40(2):378-387.

40. Ling Xue, Leslie Chiang, Astar Winoto. The role of the PI3K-AKT kinase pathway in T cell development beyond the $\beta$ checkpoint. Eur J Immunol. 2008 Nov;38(11):3200-7.

41. Hong Ji, Felix Rintelen, Caroline Waltzinger. Inactivation of PI3K $\gamma$ and PI3Kס distorts T-cell development and causes multiple organ inflammation. Blood. 2007 Oct 15;110(8):2940-7.

42. Yanjun Jia, Qiuyun Yang, Yanping Wang. Hyperactive PI3Ko predisposes naive T cells to activation via aerobic glycolysis programs. Cell Mol Immunol. 2020 Feb 25.

43. Puel A, Sophie Cypowyj, László Maródi. Inborn errors of human IL-17 immunity underlie chronic mucocutaneous candidiasis. Curr Opin Allergy Clin Immunol. 2012; 12(6):616-622.

44. Sonoko Sakata, Miyuki Tsumura, Tadashi Matsubayashi. Autosomal recessive complete STAT1 deficiency caused by compound heterozygous intronic mutations. Int Immunol. 2020 Sep 30;32(10):663-671. 
45. M Dasouki, A Jabr, G AlDakheel . TREC and KREC profiling as a representative of Thymus and Bone Marrow output in patients with various Inborn Errors of Immunity. Clin Exp Immunol. 2020 Oct;202(1):6071.

46. Julie Toubiana, Satoshi Okada, Julia Hiller. Heterozygous STAT1 gain-of-function mutations underlie an unexpectedly broad clinical phenotype. Blood. 2016 Jun 23;127(25):3154-64.

47. Xuemei Chen, Qiling Xu, Xiaolin Li. Molecular and Phenotypic Characterization of Nine Patients with STAT1 GOF Mutations in China. J Clin Immunol. 2020 Jan;40(1):82-95.

48. Giuliana Lama, Ilaria Luongo, Giuseppina Tirino. T-Lymphocyte Populations and Cytokines in Childhood Nephrotic Syndrome. Am J Kidney Dis. 2002 May; 39(5): 958-65.

49. Daniel V, Y Trautmann, M Konrad. T-Lymphocyte populations, cytokines and other growth factors in serum and urine of children with idiopathic nephrotic syndrome. Clin Nephrol.1997 May; 47(5):289-97.

50. Mackall CL, L Granger, M A Sheard. T-cell regeneration after bone marrow transplantation: differential CD45 isoform expression on thymic-derived versus thymic-independent progeny. Blood. 1993 Oct $15 ; 82(8): 2585-94$.

51. Kenneth Weinberg, B R Blazar, J E Wagner. Factors affecting thymic function after allogeneic hematopoieticstem cell transplantation. Blood. 2001 Mar 1;97(5):1458-66.

52. Andrew R. Gennery. Predicting the future with TRECs. Blood. 2018 Oct 25; 132 (17):1731-1733.

\section{Hosted file}

figure QinZhao.pdf available at https://authorea.com/users/390192/articles/504602-trendsin-trecs-values-according-to-age-and-gender-in-chinese-children-and-their-clinicalapplication

\section{Hosted file}

Figure legend and tables QinZhao.pdf available at https://authorea.com/users/390192/articles/ 504602-trends-in-trecs-values-according-to-age-and-gender-in-chinese-children-and-theirclinical-application 